AUSTRALIAN JOURNAL OF BASIC AND
APPLIED SCIENCES
EISSN: $2309-8414$

\title{
Study of Some Electrical Characteristics of the Cylindrical Plasma Jet Using Argon Gas
}

\author{
${ }^{1}$ Mohamed A. Hassouba, ${ }^{2}$ Abdel-Aleam H. Mohamed, ${ }^{1}$ Hala I. El- Naggar \\ ${ }^{1}$ Plasma Physics, Professor, Department of Physics, Faculty of Science, Benha University, Benha, Egypt, hassouba@yahoo.com \\ ${ }^{2}$ Abdel-Aleam H. Mohamed, Plasma Physics, Associate Professor, Department of Physics, Faculty of Science, Beni-Suef University, Beni- \\ Suef, Egypt. \\ ${ }^{I}$ Plasma Physics, Doctor, Department of Physics, Faculty of Science, Benha University, Benha, Egypt, and Associate Professor, Department \\ of Physics, Faculty of Sciences and Arts, Bisha University, Al-Namas, KSA.
}

Address For Correspondence:

Hala I. El- Naggar, Bisha University, Department of Physics, Faculty of Sciences and Arts, P.O. Box 101 Al-Namas 61977, KSA. Email: halaalnaggar@yahoo.com

\section{ARTICLE INFO}

\section{Article history:}

Received 12 October 2017

Accepted 22 December 2017

Available online 31 December 2017

Keywords:

Argon plasma jet, waveforms, plasma lengths, photos of plasma, power efficiency

\begin{abstract}
A B S T RA C T
BACKGROUND: Plasma jets have several advantages compared to volume or surface dielectric barrier discharges particularly concerning the enhanced control of discharge conditions and reactive species output. However, a feed gas has to be supplied for the operability of plasma jets. Commonly noble gases are used for this purpose. The interest in these atmospheric pressure plasmas have been emphasized in practical applications because it minimizes the need for vacuum systems and enables the treatment of materials continuously. OBJECTIVE: The aim of the present work was the study of some of the electrical characteristics of the atmospheric plasma jet and determine some of its parameters. We investigate some of the electrical characteristics of the atmospheric argon plasma jet which were applied for the surface modification. RESULTS: The waveforms of the applied voltage and current across the two electrodes of the plasma jet as a function of argon gas flow rate are measured. An adjustable high voltage power supply with the amplitude from 0 to $30 \mathrm{kV}$ and the frequency from 20 to $50 \mathrm{kHz}$ is connected to the upper electrode, and the bottom electrode is grounded. The plasma lengths in the plasma jet at different experimental conditions are calculated using photos of the plasma column at different discharge gas flow rate and at different discharge voltage. The deposited and consumed power and power efficiency were calculated. CONCLUSION: The results indicate that the gas flow plays a key role in variation of the structure and contributes much to enlarging the plasma lengths. The maximum values of plasma length are dependent on the values of applied voltage.
\end{abstract}

\section{INTRODUCTION}

Glow discharge can be occurred at either atmospheric pressure or low pressure. Since the last century, many research projects on glow discharge at low pressure were developed and it is well established. It is widely used in many applications such as surface treatment, light sources and thin film coating. Yet, it is costly in order to maintain at low-pressure condition. Due to this limitation, many researches had been implemented on stabilization of glow plasma at atmospheric pressure (Kanazawa et al., 1988; Yokoyama et al., 1990). Several researchers claimed that glow plasma is possible to stabilize at atmospheric pressure if three simple requirements are fulfilled: (i) source of frequency is over $1 \mathrm{kHz}$, (ii) dielectric plate is inserted between the two metal electrodes, (iii) helium is used as a dilution gas (Harry and Yahya, 1999).

Another important result relating to the stabilization of an atmospheric pressure glow discharge (APGD) is its appearance in air, argon and oxygen when using a $50 \mathrm{~Hz}$ source and a fine wire mesh as the discharge electrodes (Okazaki et al., 1993). These initial experiments have since been confirmed (Yokoyama et al., 1990;

Open Access Journal

Published BY AENSI Publication

(C) 2017 AENSI Publisher All rights reserved

This work is licensed under the Creative Commons Attribution International License (CC BY).

http://creativecommons.org/licenses/by/4.0/

\section{(c) (i) Open Access}

To Cite This Article: Mohamed A. Hassouba, Abdel-Aleam H. Mohamed, Hala I. El- Naggar, Study of Some Electrical Characteristics of the Cylindrical Plasma Jet Using Argon Gas. Aust. J. Basic \& Appl. Sci., 11(15): 26-33, 2017 
Tepper and Lindmyer, 2000), and it has also been established that fine mesh electrodes produce a more stable glow than do coarse mesh electrodes (Tepper et al., 2002). Since ozone is commonly generated in air or in oxygen, the production of an APGD in air is potentially important, and forms therefore the focus of the present investigation.

Besides reducing the characteristic length of the discharge chamber, stable atmospheric pressure glow discharges (APGDs) used for technological applications have also been operated when other conditions are fulfilled with respect to the structure of the electrodes, the carrier gas and the frequency of the applied voltage (Francis, 1956; Bogaerts et al., 2002). Typically, in APGDs, at least one of the electrodes is covered with a dielectric, and the discharge operates at alternating voltages. Furthermore, the kind of discharge gas determines the stability of the glow discharge.

The main advantage of APGDs is the absence of vacuum conditions, which greatly reduces the cost and complexity of the glow discharge operation (Deng et al., 2005). This generic capability has brought in the last ten years much closer to practical applications such as biological decontamination and open -air surface coating (Shi et al., 2006).

Plasma treatments are realized by the plasma plume, i.e. the plasma developing at the outlet of the discharge reactor flushed with rare gas expanding in the ambient air. Plasma plume length and gas jet physics are coupled as already mentioned in literature (Karakas et al., 2010). Plasma plume length depends on several parameters such as pulse width, applied voltage, and also the gas flow (Jiang et al., 2011).

The effects of air diffusion on the spatial distribution of $O H$ radicals and $O$ atoms in the afterglow of atmospheric-pressure plasma jets was studied (Nikiforov et al., 2014). These effects are investigated by performing and comparing measurements in ambient air with measurements in a controlled argon atmosphere without the admixture of air, for an argon plasma jet. The higher density of active species in the discharge in ambient air is likely due to a higher electron density and a more effective electron impact dissociation of $\mathrm{H}_{2} \mathrm{O}$ and $\mathrm{O}_{2}$ caused by the increasing electrical field, when the discharge is operated in ambient air.

Arrays of atmospheric-pressure plasma jets as a means to increase the area being treated in surface modification and in plasma medicine in particular was considered (Babaeva and Kushner 2014). A unique challenge of scaling plasma jet arrays is that individual plasma jets in an array tend to interact with each other, which can lead to quenching of some individual jets. To investigate these potential interactions, a computational study of one-, two- and three-tube arrays of micro-plasma jet arrays was performed. Ionization waves (IWs) or plasma bullets, which form the jets on the boundaries of an array, encounter higher mole fractions of air earlier compared with the center jet and so are slower or are quenched earlier. The close proximity of the jets produces electrostatic repulsion, which affects the trajectories of the IWs. If the jets are close enough, photo ionizing radiation from their neighbors is an additional form of interaction.

The effects of the gas flow on the propagation of atmospheric pressure plasma plumes within the range of the Ar flow rates from 2 to $6 \mathrm{l} / \mathrm{min}$, was studied (Wu et al., 2013). The length of the downstream plasma plume was found larger compared to the upstream plume. Moreover, the length difference $L_{\text {down }}-L_{u p}$ became larger with the increase of the gas flow rate.

The effect of gas flow rate of helium and argon on the length of dielectric barrier discharge (DBD) jet generated under atmospheric pressure using an AC source was investigated (Lau et al., 2014). It is found that as the flow rate increases, the jet length increases up to a maximum length under laminar flow. Further increase in flow rate, it will cause the jet length to decrease as the flow becomes turbulent. Also, was found that, there is an obvious increment in jet length of argon DBD system when the applied voltage is increased from $8.8 \mathrm{kV}$ to $11.0 \mathrm{kV}$, but not in helium DBD jet. The longest jet in helium is obtained at $17-19 \mathrm{1} / \mathrm{min}$ and $8.8 \mathrm{kV}$ while in argon, it is obtained at $5.5 \mathrm{l} / \mathrm{min}$ and $11.0 \mathrm{kV}$.

The characterization of electrical power distribution in an atmospheric pressure plasma jet operated at six different frequencies was studied (Gerling et al., 2017). It was found, that the effluent length of the device scales linearly with the plasma power and total input power into the effluent increases continuously with frequency.

The aim of the present work is to investigate some of the electrical characteristics of the atmospheric plasma jet and determine some of its parameters.

\section{MATERIALS AND METHODS}

A schematic diagram of the plasma jet and its power supply is shown in fig. 1. the electrical characterization of the plasma device is performed in order to gain access to the values of the dissipated power into the plasma. Established techniques are limited by the applied frequency of the plasma jet and the resulting wave nature of the physical values. Due to the frequency range used for the plasma jet under investigation, the dissipated power determination has to be adapted based on techniques introduced in the literature.

The plasma jet consists of two cylindrical electrodes; the inner electrode is made of stainless steel of width $1 \mathrm{~mm}$, enclosed by quartz tube of diameter $4 \mathrm{~mm}$. The other electrode is covered by copper tape and connected 
to the earth. An adjustable high voltage power supply with the amplitude from 0 to $30 \mathrm{kV}$ and the frequency from 20 to $50 \mathrm{kHz}$ is connected to the upper electrode, and the bottom electrode is grounded.

Electrical measurements of the applied voltage and discharge current are measured using Tektronix voltage probe (model P6015A), while the current flowing in the circuit is measured using current monitor (model 6585 Pearson electronics, USA). The applied voltage on the two electrodes and the discharge current through the reactor are monitoring using a $500 \mathrm{MHz}$ four-channel digital phosphor oscilloscope type Tektronix (model DPO 4054). Argon gas is supplied at a rate of 1.5 standard liters per minute (SLPM) using a calibrated mass flow controller and a Canon (EOS40D) camera was used to capture the image of the plasma jet.

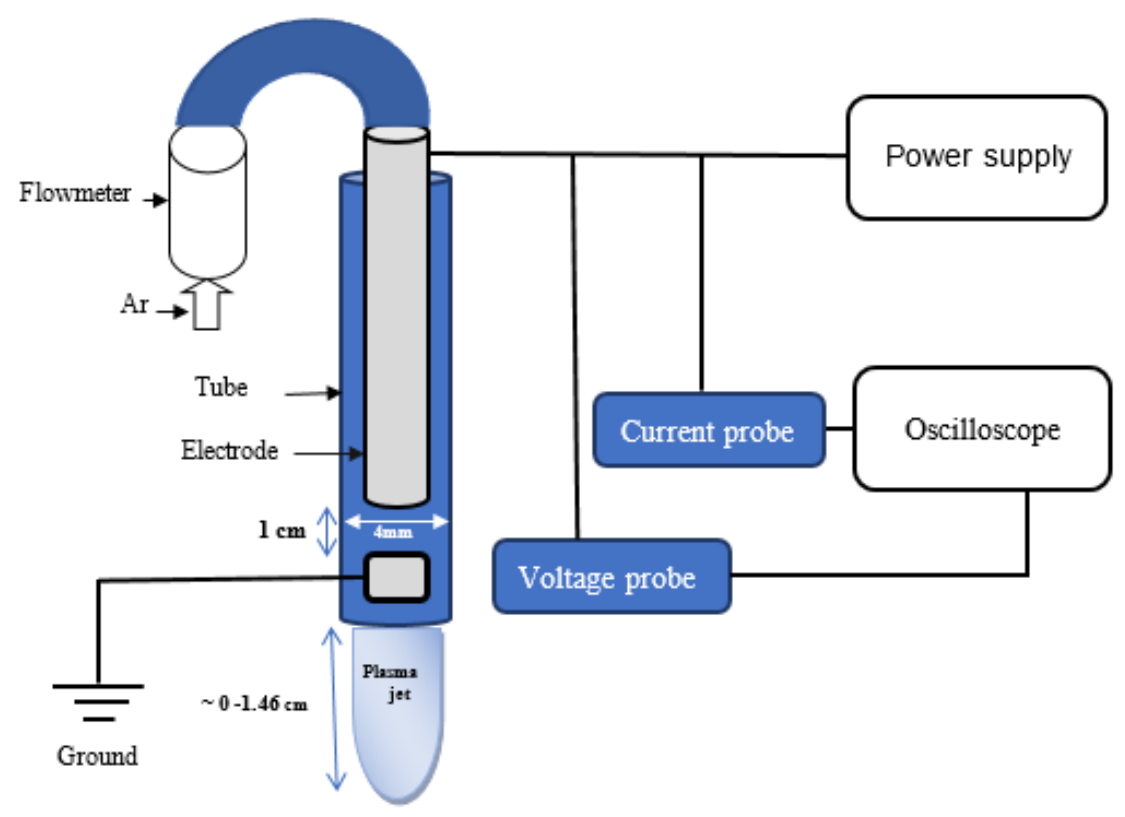

Fig. 1: Schematic diagram of the experimental setup

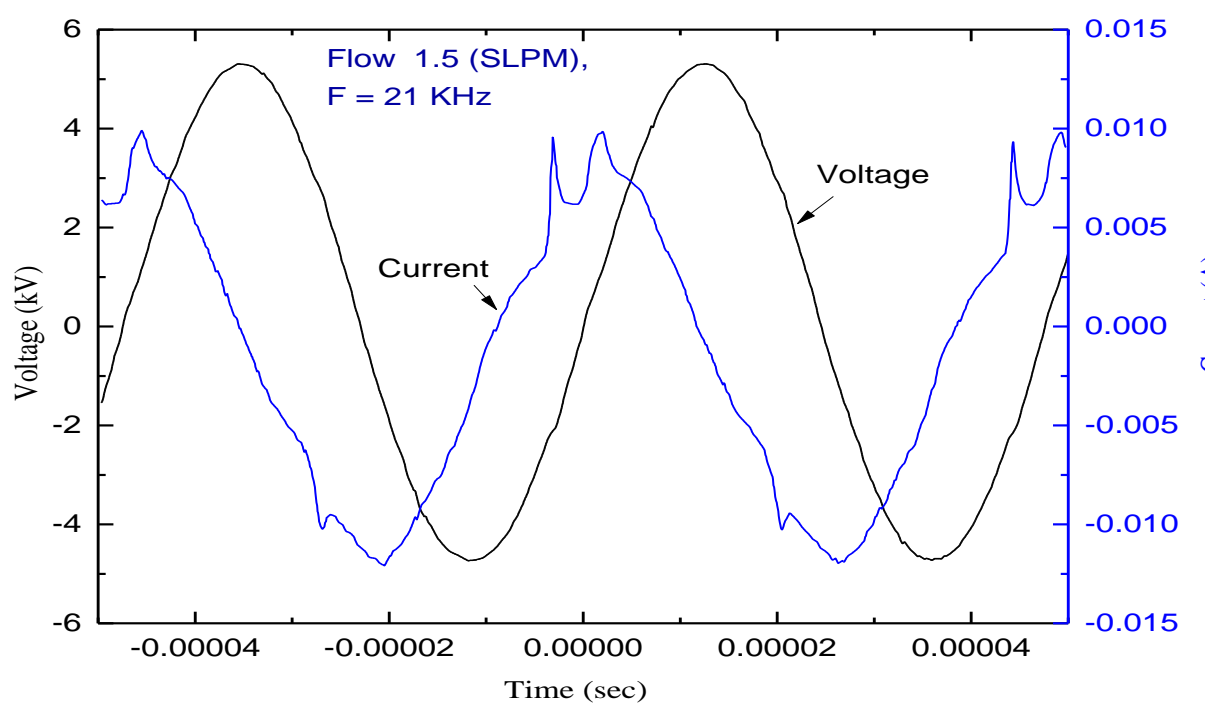

Fig. 2: V-I waveform for Ar gas at flow rate 1.5 (SLPM) and $21 \mathrm{kHz}$

\section{RESULTS AND DISCUSSION}

The waveforms of the applied voltage and current across the two electrodes of the plasma jet as a function of Ar gas flow rate are shown in figure (2) and figure (3). Spike currents are observed in the current waveform in the rising portion of each half cycle of the applied voltage from the start to the time of voltage peaking. The 
spikes are caused by the filament discharges, which compose the dielectric barrier discharge in air at atmospheric pressure (Bogaerts et al., 2002; Wu et al., 2013).

As the two electrodes are of the same size and shape, the discharge current in the positive half-cycle is about the same as that in the negative half-cycle. The APGD is character by a single current pulse per half-cycle of the applied voltage with the same periodicity.



Fig. 3: V-I waveform for Ar gas at flow rate 3.5 (SLPM) and $21 \mathrm{kHz}$

Like low-pressure glow discharges, plasma jet is characterized by relatively strong light emission near the cathode. Photos (1 and 2) show the plasma lengths intensity in the plasma jet at different experimental conditions, as shown in figure (4). From these photos we can calculate the plasma lengths at different discharge gas flow and at different discharge voltage (Lau et al., 2014).

\begin{tabular}{|l|c|c|c|c|c|c|c|}
\hline Voltage $5(\mathrm{kV})$ & Fre.21 (kHz) & flow 2.5 (SLPM) & $\begin{array}{c}\text { Plasma length 1 } \\
(\mathrm{cm})\end{array}$ & $\begin{array}{c}\text { Voltage 7 } \\
(\mathrm{kV})\end{array}$ & Fre.21 (kHz) & flow 4 (SLPM) & $\begin{array}{c}\text { Plasma length } \\
1.13(\mathrm{~cm})\end{array}$ \\
\hline & & & & \\
& & & \\
& & &
\end{tabular}

Fig. 4: Plasma lengths intensity in the plasma jet at different experimental conditions

Figure (5) and figure (6) Shows the relation between the plasma lengths, which calculated from the above, photos as a function of applied voltage at different Ar gas flow rate. It's clear from this figures that, the plasma 
lengths increase with increases the applied voltage until reaching to the saturation at higher values of applied voltages (Karakas et al., 2010).

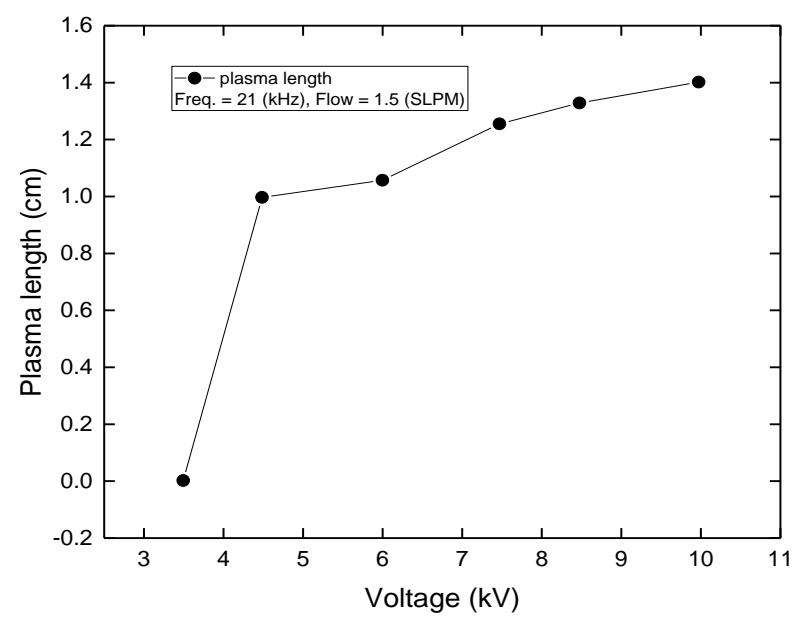

Fig. 5: Relation between Plasma length and applied voltage at Flow 1.5 (SLPM) and $21 \mathrm{kHz}$

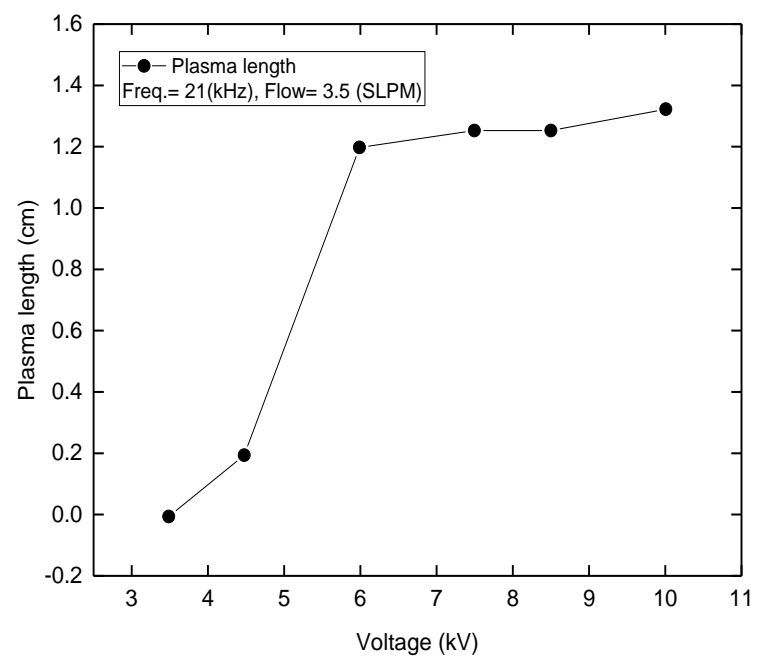

Fig. 6: Relation between Plasma length and applied voltage at Flow 3.5 (SLPM) and $21 \mathrm{kHz}$ 




Fig. 7: Relation between Plasma length and Argon flow rate at $5 \mathrm{kV}$ and $21 \mathrm{kHz}$

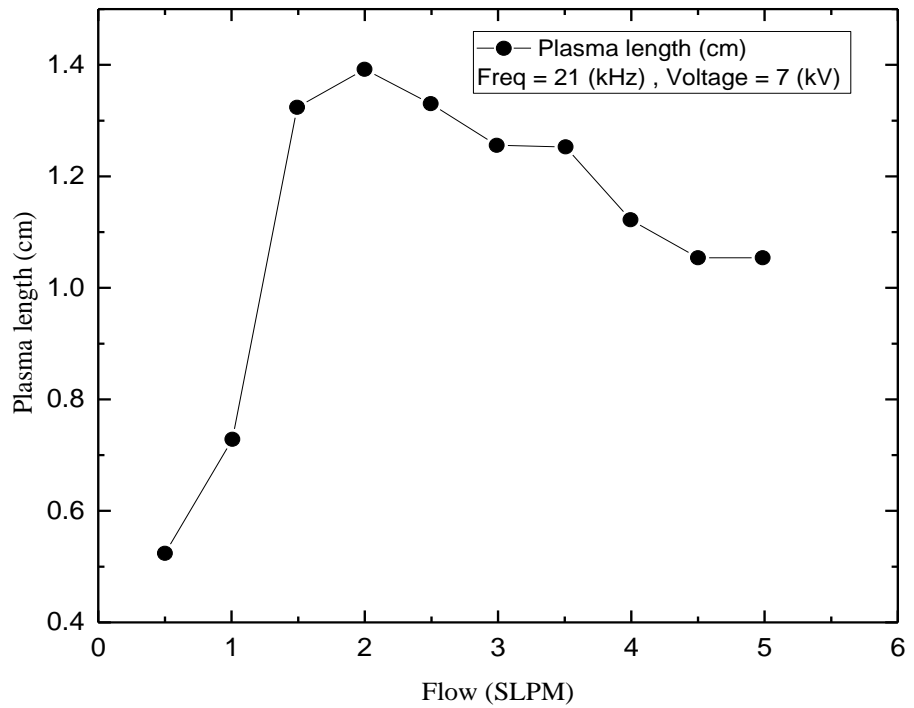

Fig. 8: Relation between Plasma length and argon flow rate at $7 \mathrm{kV}$ and $21 \mathrm{kHz}$

Figure (7) and figure (8) show the relation between the plasma length, which determined from the above photos in figure (4), as a function of argon flow rate at different applied voltage. These figures show that, the plasma length increases with increasing the flow rate until it reaches to maximum value then it decreases to reach to nearly saturation at higher flow rate (Lau et al., 2014). In the low argon flow rate region, when flow rate increases gradually, more gas particles are available to be ionized and the number of charged particles forced out from the quartz tube would be higher, so a longer plasma length is observed. This is characteristic of laminar flow (Li et al., 2009). After a maximum length of plasma jet, the length becomes shorter when flow rate is increased. This is due to turbulence flow of the gas in quartz tube (Lau et al., 2014). The maximum values of plasma length are dependent also on the values of applied voltage.

On the other hand, figure (9) shows the power deposited during one-cycle discharge of plasma jet at a frequency of $21 \mathrm{kHz}$ as a function of applied peak voltage (peak -to-peak), $\mathrm{V}_{\mathrm{pp}}$. The power linearly increases with increasing applied peak voltage (Gerling et al., 2017).

Generally, the average power of an electric signal of period $T=\frac{1}{f}$ is defined by (Dunnbier et al., 2015): $\mathrm{p}=\frac{1}{\mathrm{~T}} \int_{\mathrm{t}_{\mathrm{o}}}^{\mathrm{t}_{\mathrm{o}}+\mathrm{T}} \mathrm{I}(\mathrm{t}) \mathrm{V}(\mathrm{t}) \mathrm{dt}$

Where $\mathrm{I}(\mathrm{t})$ and $\mathrm{V}(\mathrm{t})$ being the instantaneous current and voltage respectively. In case of a sinusoidal waveform, equation (1) can be written as (Dunnbier et al., 2015):

$\mathrm{p}=\mathrm{I}_{\mathrm{RMS}} \mathrm{V}_{\mathrm{RMS}} \cos (\varphi)$ 
Where $I_{R M S}=I_{\circ} / \sqrt{2}$ and $V_{R M S}=V_{\circ} / \sqrt{2}$ are the root mean square (RMS) current and voltage, respectively. $I_{\circ}$ and $V_{\circ}$ are the peak values of the current and voltage respectively and $\varphi$ is the phase angle between the discharge voltage and current. Usually, the total power (power deposited) delivered by the generator into the electrical network down to the plasma can be calculated from measurements of current, voltage and phase angle $\varphi$ directly at the electrodes.

Moreover, the consumed power $\mathrm{P}$ is obtained using equation (1) and its calculated value is equal to $23.2 \mathrm{~W}$. Also, the mean energy consumed is estimated by multiplying the consumed power by the period time, which gives $2.3 \mathrm{~mJ}$ (Dunnbier et al., 2015).

The power efficiency of the plasma jet device can be calculated by dividing the measured consumed power by the input power to be $58 \%$, (Input power for this system is equal to $40 \mathrm{~W}$ ).

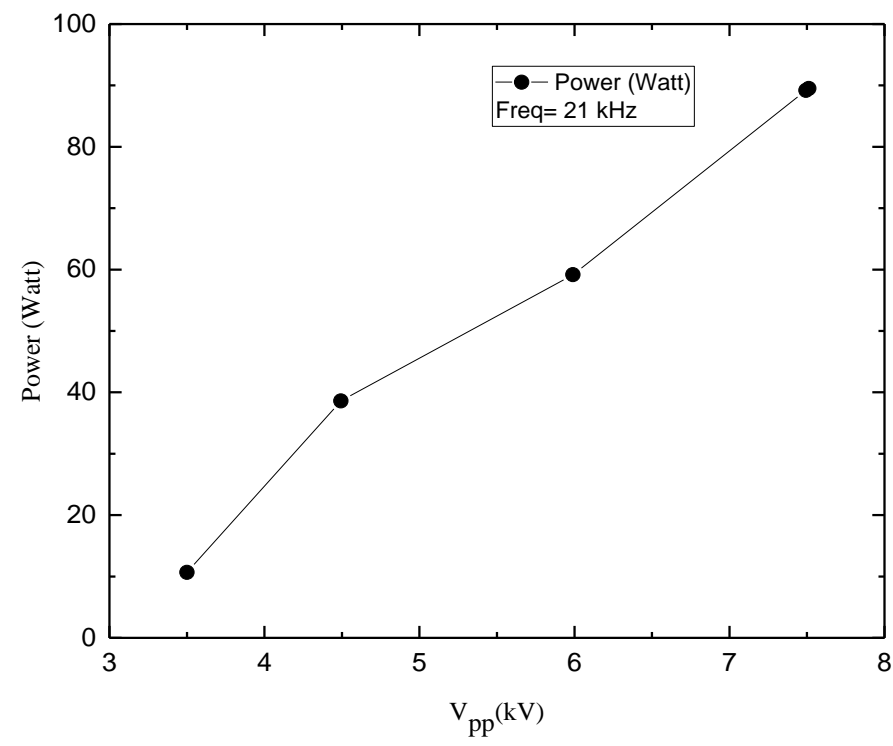

Fig. 9: Relation between power deposited during one- cycle discharge and applied peak voltage at $21 \mathrm{kHz}$

From the above results, one could be clarified that, the gas flow rate is one of the important parameters that can affect the plasma jet length. The role of the gas flow rate and different experimental conditions such as applied voltage and frequency in atmospheric pressure plasma jets can be used in improving control of this type of non-equilibrium plasmas as in plasma biology, health care, medicine, surface modification, and nanotechnology applications.

\section{REFERENCES}

Babaeva, Yu Natalia \& Kushner, J. Mark, 2014. Interaction of Multiple Atmospheric-Pressure MicroPlasma Jets in Small Arrays: He/O2 into Humid Air. Plasma Sources Science and Technology, $23(1): 015007$.

Bogaerts, A., E. Neyts, R. Gijbels, J. Van der Mullen, 2002. Gas Discharge Plasmas and Their Applications. Spectrochimica Acta part B 57: 609-658.

Deng, T.X., J.J. Shi, G. Shama, M.G. Kong, 2005. Effects of Microbial Loading and Sporulation Temperature on Atmospheric Plasma Inactivation of Bacillus Subtilis Spores. Applied Physics Letters 87: 153901.

Dunnbier, M., M.M. Becker, S. Iseni, R. Bansemer, D. Loffhagen, S. Reuter, KD. Weltmann, 2015. Stability and Excitation Dynamics of an Argon Micro-Scaled Atmospheric Pressure Plasma Jet. Plasma Sources Science and Technology 24(6): 065018.

Francis, G., 1956. The Glow Discharge at Low Pressure, in: S. Flugge (Ed.), Handbuch der Physik, 22: Springer“ -Verlag, Berlin.

Gerlinga, T., R. Brandenburg, C. Christian Wilke, K.D. Weltmann, 2017. Power measurement for an atmospheric pressure plasma jet at different frequencies: distribution in the core plasma and the effluent. The European Physical Journal Applied Physics, 78: 10801.

Harry, J.E., A.A. Yahya, 1999. Factors Affecting the Glow -to- Arc Transition at the Cathode of an Electric Discharge at Atmospheric Pressure. International Journal of Electronics Electronics, 86(6): 755-762.

Jiang, N., J.L. Yang, F. He, Z. Cao, 2011. Interplay of Discharge and Gas Flow in Atmospheric Pressure Plasma Jets. Journal of Applied Physics 109: 093305. 
Kanazawa, S., M. Kogoma, T. Moriwaki, S. Okazaki, 1988. Stable Glow Plasma at Atmospheric Pressure. Journal of Physics D: Applied Physics, 21(5): 838-840.

Karakas, E., M. Koklu, M. Laroussi, 2010. Correlation between Helium Mole Fraction and Plasma Bullet Propagation in Low Temperature Plasma Jets. Journal of Physics D:Applied Physics, 43(15): 155202.

Lau, Y.T., K.K. Jayapalan, M.E. Pam, O.H. Chin, 2014Dependence of Dielectric Barrier Discharge Jet Length on Gas Flow Rate and Applied Voltage. Journal of Science and Technology in the Tropics, 10(2): 131138.

Li, Q., J.T. Li, W.C. Zhu, X.M. Zhu, Y.K. Pu, 2009. Effects of Gas Flow Rate on the Length of Atmospheric Pressure Nonequilibrium Plasma Jets. Applied Physics Letters, 95:141502.

Nikiforov, A., L. Li, N. Britun, R. Snyders, P. Vanraes, C. Leys, 2014. Influence of Air Diffusion on the $\mathrm{OH}$ Radicals and Atomic O Distribution in an Atmospheric Ar (bio) Plasma Jet. Plasma Sources Science and Technology, 23(1): 015015.

Okazaki, S., M. Kogoma, M. Uehara, Y. Kimura, 1993. Appearance of Stable Glow Discharge in Air, Argon, Oxygen and Nitrogen at Atmospheric Pressure Using $50 \mathrm{~Hz}$ Source. Journal of Physics D: Applied Physics, 26(5): 889-892.

Shi, J.J., D.W. Liu, M.G. Kong, 2006. Plasma Stability Control Using Dielectric Barriers in RadioFrequency Atmospheric Pressure Glow Discharges. Applied Physics Letters, 89: 081502.

Tepper, J., M. Lindmayer, 2000. Investigations on Two Different Kinds of Homogeneous Barrier Discharges at Atmospheric Pressure. Proc. of Int. Symp. on High pressure. Low Temperature Plasma Chemistry, Hakone VII, Greifswald, Germany, pp: 38-43.

Tepper, J., P. Li, M. Lindmayer, 2002. Effects of Interface Between Dielectric Barrier and Electrode on Homogeneous Barrier Discharges at Atmospheric Pressure. XIV International Conference on Gas Discharges and their Application, Liverpool, United Kingdom, pp: 1-4.

Wu, S., Z. Wang, Q. Huang, X. Tan, X. Lu, K. Ostrikov, 2013. Atmospheric-Pressure Plasma Jets: Effect of Gas Flow, Active Species, and Snake-Like Bullet Propagation. Physics of Plasmas, 20: 023503.

Yokoyama, T., M. Kogoma, T. Moriwaki, S. Okazaki, 1990The Mechanism of the Stabilization of Glow Plasma at Atmospheric Pressure. Journal of Physics D: Applied Physics, 23(8): 1125-1128. 